\title{
ASCA OBSERVATIONS OF THE SUPERLUMINAL JET SOURCE GRS1915+105
}

KEN EBISAWA, T. TAKESHIMA, N. E. WHITE

Laboratory for High Energy Astrophysics, NASA/GSFC

Greenbelt, MD, 20771, USA

T. KOTANI

The Institute of Physical and Chemical Research (RIKEN)

2-1 Hirosawa, Wako, Saitama, 351-01, Japan

T. DOTANI, Y. UEDA

Institute of Space and Astronautical Science

Yoshinodai, Sagamihara, Kanagawa, 229, Japan

B. A. HARMON, C. R. ROBINSON, S. N. ZHANG $N A S A / M S F C$

Huntsville, AL 35806, USA

W. S. PACIESAS

Department of Physics, University of Alabama in Huntsville Huntsville, AL 35899, USA

M. TAVANI

Columbia Astrophysics Laboratory, Columbia University New York, NY 10027, USA

AND

R. FOSTER

Naval Research Laboratory

Washington C. C. 20375, USA

\section{Introduction}

GRS1915+105 is an extraordinary X-ray transient which exhibits superluminal radio jets. In this paper, ASCA observations of the GRS1915+105 conducted from 1994 to 1997 are reported. Observations are carried out on the following dates each for $\sim 20$ ksec exposure; Sep 27 1994, April 20 1995, Oct 231996 and Apr 251997. 

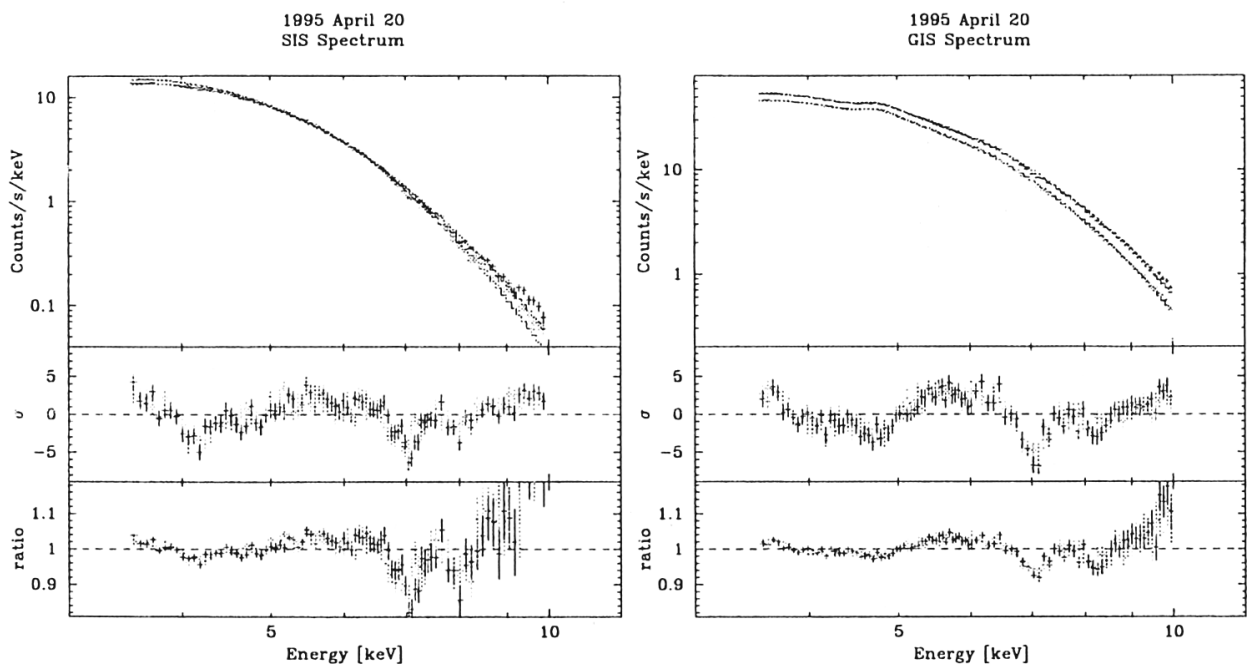

Figure 1. SIS (left) and GIS (right) energy spectra in April 1995 fitted with an absorbed cut-off power-law model. Absorption line features are clearly seen in the residuals.

\section{Results}

Main results may be summarized as follows:

1. The source is highly variable at various time scales, and in particular characteristic burst/dip structures were observed in October 1996 and April 1997. The flux variation is larger above $3 \mathrm{keV}$, and the spectral change is most simply described by the reduction of the cut-off energy when the X-ray flux becomes lower.

2. The continuum energy spectra are approximately fitted by an absorbed cut-off power-law with the form $A E^{-\alpha} e^{-E / E_{c}}$. Overall, there is not a clear correlation between the cut-off energy and the X-ray flux. The hydrogen column densities are always within the range of $3.5-4.1$ $\times 10^{22} \mathrm{~cm}^{-2}$.

3. Characteristic absorption line features are seen in September 1994 and April 1995 at $\sim 6.7 \mathrm{keV}, 7.0 \mathrm{keV}$ and $8.0 \mathrm{keV}$ (figure 1). The former two may be identified as the $K \alpha$ lines from He-like and $\mathrm{H}$-like irons and the last one may be the $K \beta$ line from He-like iron. These features are vaguely seen in October 1996, and in April 1997, an emission linelike feature is seen at $\sim 6.7 \mathrm{keV}$. These absorption line features are reminiscent of the similar spectral features in the other Galactic superluminal jet source GRO J1655-40 (Ueda et al. 1997, ApJ Letter, in print).

The postscript file of the poster paper presented at the conference can be found at ftp://lheaftp.gsfc.nasa.gov/pub/ebisawa/kyoto.ps.gz. 